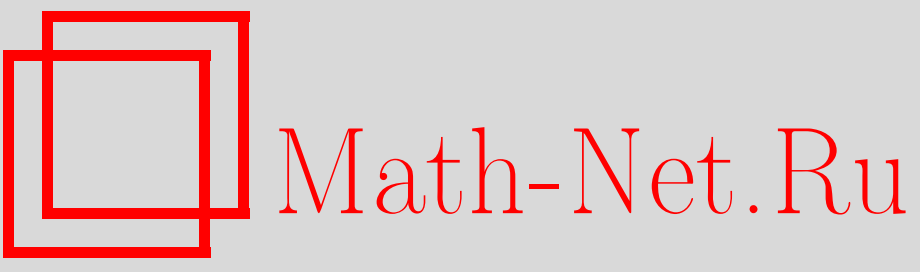

А. А. Туганбаев, Дистрибутивные нелокализуемые кольца, УМН, 2002, том 57, выпуск 3, 165-166

DOI: https://doi.org/10.4213/rm530

Использование Общероссийского математического портала Math-Net.Ru подразумевает, что вы прочитали и согласны с пользовательским соглашением

http://www.mathnet.ru/rus/agreement

Параметры загрузки:

IP : 54.197 .217 .227

26 апреля 2023 г., $17: 14: 08$ 


\section{ДИСТРИБУТИВНЫЕ НЕЛОКАЛИЗУЕМЫЕ КОЛЬЦА}

\section{А. А. ТУГАНБАЕВ}

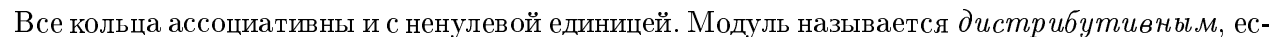
ли решетка всех его подмодулей дистрибутивна. Модуль, у которого все конечно порожденные подмодули цикличны, назьвается модулем Безу. Модуль назьвается цепныцм, если любые два его подмодуля сравнимы по включению. Модуль называется равномерным, если любые два его ненулевых подмодуля имеют ненулевое пересечение. Кольо назьвается инвариантным справ $a$, если всеего правые идеалы являются идеалами. Кольц $A$ назьвается когерентныцм справа, если каждый его конечно порожденный правый идеал - конечно представимый $A$-модуль. Слова типа "дистрибутивное кольцо" означают, что соответствующие условия выполнены справа и слева. Для кольца $A$ через $J(A), N_{\ell}(A), N_{r}(A), \max \left(A_{A}\right)$ и $\max \left({ }_{A} A\right)$ обозначаются радикал Джекобсона, множество всех левых делителей нуля, множество всех правых делителей нуля, множество всех максимальных правых идеалов и множество всех максимальных левых идеалов соответственно. Кольцо $A$ назьвается локализуемым справа, если для любого $M \in \max \left(A_{A}\right)$ существует правая локализация $A_{M}$ (т.е. существуют такое кольцо $A_{M}$ и колшцевой гомоморфизм $f: A \rightarrow A_{M}$, что все элементы из $f(A \backslash M)$ обратимы в $A_{M}, A_{M}=\left\{f(a) f(t)^{-1} \mid a \in A\right.$, $t \in A \backslash M\}$ и $\left.\operatorname{Ker}(f)=\left\{a \in A \mid(A \backslash M) \cap r_{A}(a) \neq \varnothing\right\}\right)$.

Теорема. Существует инвариантное дистрибутивное равномерное когерентное подпрямо неразложимое кольцо Безу А, которое не является локализуемым справа или слева и обладает следующими свойствами:

(1) в кольце $A$ все регулярные әлементы обратимы, пересечение любых двух конечно порожденных правых (левых) идеалов кольца $A$ является главныц правым (левым) идеалом, правый (левый) аннулятор любого әлемента является главным правым (левым) идеалом и А не изоморфно подкольцу локального кольца;

(2) $A$ содержит такой идеал $M$, что $M^{2}=0, A / M$ - коммутативная область главных идеалов, имеющая ровно два максимальных идеала, $M$ - чепной артинов делимый $A$-модуль, $M=а M=M a \subset a A=A a$ для каждого $a \in A \backslash M$ и $M$ - единственный неглавный правый или левый идеал кольца $A$;

(3) $\max \left(A_{A}\right)=\max \left({ }_{A} A\right)=\left\{N_{\ell}(A), N_{r}(A)\right\}, A / N_{\ell}(A)$ u $A / N_{r}(A)-$ nоля, $A / J(A) \cong$ $A / N_{\ell}(A) \times A / N_{r}(A), N_{\ell}(A) \cap N_{r}(A)=J(A), N_{r}(A)$ - единственный собств енныцй точный справа идеал и $N_{\ell}(A)$ - единственный собственный точный слева идеал кольца $A$.

Первый пример дистрибутивного колца, не являющегося локализуемым справа или слева, был недавно построен $\Gamma$.Е. Пунинским. Элементы из $A \backslash\left(N_{r}(A) \cup N_{\ell}(A)\right)$ назьваются регулярныли әлементами (в $A$ ). Модуль $M_{A}$ назьвается делимым, если для любого $m \in M$ и каждого регулярного $a \in A$ существует такое $x \in M$, что $m=x a$. Подмножество $T$ кольца $A$ называется реверсивным справа, если для любых таких $a \in A$ и $t \in T$, что $t a=0$, существует такое $u \in T$, что $a u=0$.

ДОКАЗАТЕЛЬСТВо тЕОРемЫ. Пусть $\mathbb{Z}$ - колью целых чисел, $\mathbb{Q}$ - поле рациональных чисел, $\mathbb{Q}[i]$ - поле частных кольца целых гауссовых чисел $\mathbb{Z}[i]\left(i^{2}=-1\right), \varphi$ - такой автоморфизм поля $\mathbb{Q}[i]$, что $q_{1}+q_{2} i \rightarrow q_{1}-q_{2} i, R_{1}$ и $R_{2}$ - локализации коммутативной области главных идеалов $\mathbb{Z}[i]$ по простым идеалам, порожденным элементами $2+i$ и $2-i$ соответственно. Тогда $R_{1}$ и $R_{2}$ - коммутативные цепные области главных идеалов. Пусть $R \equiv R_{1} \cap R_{2}$ и $X, Y$ - идеалы кольца $R$, порожденные элементами $2+i$ и $2-i$ соответственно. Коммутативная область главных идеалов $R$ имеет ровно два максимальных идеала $X$ и $Y, R_{X}=R_{1}, R_{Y}=R_{2}, \varphi\left(R_{X}\right)=R_{Y}$, $X+Y=R, J(R)=X \cap Y=(2+i)(2-i) R=5 R$ и фактор-колшцо $R / J(R)$ изоморфно прямому произведению полей $A / X$ и $A / Y$. Кольцо $R$ совпадает с множеством всех несократимых рациональных гауссовых дробей, знаменатели которых не делятся ни на $2+i$, ни на $2-i$. Пусть $M$ - правый $R$-модуль $\mathbb{Q}[i] / R_{X}$. Все собственные подмодули в $M_{R}$ цикличны и образуют бесконечную счетную строго возрастающую цепь $0=s_{0} R \subset s_{1} R \subset s_{2} R \subset \cdots$, где простой модуль $s_{1} R$ изоморфен $R / Y=R /(2-i) R$ и $r_{R}\left(s_{n}\right)=(2-i)^{n} R$ для всех натуральных $n$.

Работа выполнена при поддержке Российского фонда фундаментальных исследований (грант № 99-01-00382). 
Мы также превратим $M$ в левый $R$-модуль по правилу $r m=m \varphi(r)$ для всех элементов $r \in R$ и $m \in M$. Тогда $M-(R, R)$-бимодуль, являющийся цепньм артиновым делимьм правым (левьпм $R$-модулем. Пусть $A$ - внешняя прямая сумма абелевых групп $R$ и $M$ с таким умножением, что $\left(r_{1}, m_{1}\right)\left(r_{2}, m_{2}\right)=\left(r_{1} r_{2}, r_{1} m_{2}+m_{1} r_{2}\right)=\left(r_{1} r_{2}, m_{2} \varphi\left(r_{1}\right)+m_{1} r_{2}\right)$ для всех $r_{1}, r_{2} \in R$ и $m_{1}, m_{2} \in M$. Пара $(1,0)$ является единицей колшца $A$. Мы отождествляем $M, X$ и $Y$ с идеалами $(0, M),(X, 0)$ и $(Y, 0)$ колњца $A$ соответственно. Колњцо $R$ отождествляется с подколшцом $(R, 0)$ в $A$. Правые (левые) идеалы колшца $A$, лежащие в $M$, совпадают с $R$-подмодулями в $M$, $m A=A m$ для любого $m \in M, A / J(A)$ изоморфно прямому произведению полей $A / X$ и $A / Y$, $M^{2}=0, A / M$ изоморфно коммутативной области главных идеалов $R, M$ - цепной артинов делимый правый (левый) $A$-модуль Безу, простой правый (левьй) $A$-модуль $s_{1} A$ совпадает с $A s_{1}$ и является наименьшим ненулевым правым (левым) идеалом кольца $A, r_{A}\left(s_{1}\right)=Y$ и $\ell_{A}\left(s_{1}\right)=X$. Приведем некоторые свойства кольца $A$.

(1) $X=(2+i) A=\ell_{A}\left(s_{1}\right)=N_{\ell}(A) \in \max \left(A_{A}\right)=\max \left({ }_{A} A\right)$ и $Y=(2-i) A=r_{A}\left(s_{1}\right)=$ $N_{r}(A) \in \max \left(A_{A}\right)=\max \left({ }_{A} A\right), \max \left(A_{A}\right)=\max \left({ }_{A} A\right)=\left\{N_{\ell}(A), N_{r}(A)\right\}, N_{r}(A)$ - единственный собственньй точный справа правый (левый) идеал кольца $A$ и $N_{\ell}(A)$ - единственный точньй слева собственньй правый (левый) идеал кольца $A$.

(2) Элемент $(2+i)+(2-i)=4=5-1$ обратим в $A$, так как $5 \in J(A)$. Если $x \equiv(2+i) / 4 \in X$, то $1-x=(2-i) / 4 \in Y,(2+i) s_{1}=s_{1} \varphi(2+i)=s_{1}(2-i)=0, x s_{1}=s_{1}(1-x)=0$.

(3) $x \in X \backslash J(A)=X \backslash Y \subseteq N_{\ell}(A), 1-x \in Y \backslash X=Y \backslash J(A) \subseteq N_{r}(A), J(A)$ содержит не все правые (левые) делители нуля кольца $A$, правый (левый) аннулятор любого элемента из $A-$ главньй правый (левый) идеал.

(4) $A / M$ - коммутативная область главных идеалов, цепной идеал $M$ кольца $A$ сравним по включению с любым правым или левым идеалом из $A, s_{1} A=A s_{1}=r(X)=\ell(Y)$ - наименьший ненулевой правый (левый) идеал кольца $A$ и $A \cong A / r(X)=A / \ell(Y)$.

(5) $A$ - инвариантное дистрибутивное равномерное подпрямо неразложимое кольцо Безу, все регулярные элементы кольца $A$ обратимы в $A$ и пересечение любых двух конечно порожденных правых (левых) идеалов колшца $A$ - главный правый (левый) идеал.

(6) $A$-когерентное колњо, не изоморфное подколцу локального колњца.

(7) Множество $A \backslash X$ не реверсивно слева, множество $A \backslash Y$ не реверсивно справа и $A$ не является локализуемым справа или слева кольцом.

Свойства (1)-(3) проверяются непосредственно.

(4) Так как $A / M \cong R$, то $A / M$ - коммутативная область главных идеалов. Так как $M-$ делимый $R$-модуль, то $M$ сравним по включению с любым правым или левым идеалом из $A$. Тогда $s_{1} A=A s_{1}$ - наименьший ненулевой правый (левьй) идеал, поскольку $s_{1} R=R s_{1}$ - наименьший подмодуль цепного артинова правого (левого) $R$-модуля $M$. Непосредственно проверяется, что $s_{1} A=A s_{1}=r(X)=\ell(Y)$ и $A \cong A / r(X)=A / \ell(Y)$.

(5) Так как $M-A$-модуль Безу и $m A=A m$ для каждого $m \in M$, то по (4) $A$ - инвариантное подпрямо неразложимое кольцо Безу. Тогда $A$ равномерно. Инвариантное кольцо Безу $A$ дистрибутивно по теореме 3.26 из [1]. Пусть $t$-регулярный элемент. По (1) $t$ не содержится ни в каком максимальном правом или левом идеале. Поэтому $t A=A t=A$. Последнее утверждение следует из (4).

(6) Из (5), (3) и $[1 ; 7.2]$ следует, что $A$ когерентно. Допустим, что существует такое локальное кольцо $H$, что $A$ - подкольцо в $H$. По (3) $x$ и $1-x$ не регулярны. Поэтому $x$ и $1-x$ не обратимы в локальном кольце $H$; получено противоречие.

(7) По (3) $x \in A \backslash Y$. По (2) $x s_{1}=0$. Так как $r\left(s_{1}\right) \cap(A \backslash Y)=Y \cap(A \backslash Y)=\varnothing$, то $A \backslash Y$ не реверсивно справа. Аналогично, $A \backslash X$ не реверсивно слева. Если $A$ локализуемо справа, то по предложению 5.17 из [1] для любого максимального правого идеала $M$ множество $A \backslash M$ реверсивно справа.

Из приведенных выше рассуждений и свойств вытекает, что $A$ - искомое кольцо.

\section{СПИСОК ЛИТЕРАТУРЫ}

[1] A. A. Tuganbaev. Semidistributive Modules and Rings. Dordrecht: Kluwer, 1998. 